absorptiometry (DXA) was used to measure femoral neck and spine L2-4 BMD adjusted for age, weight and height. BMD was expressed in $\mathrm{g} / \mathrm{cm}^{2}$. To investigate associations between history of N-VF and disease related variables, we used bi-variate (Students t-test, Pearson Chi square test) and multivariate statistical analyses (logistic regression).

Results A total of 25 patients (Oslo 11, Truro 1 and Amsterdam 13 patients) reported a history of previous N-VF (total number of fractures 34). In the Truro patients BMD (adjusted for age, weight and height) in both spine and hip was higher than in Oslo and Amsterdam patients. A statistical difference between those with and without a previous N-VF was found for the variables (mean (SD)) listed in the Table 1 and for disease duration (22.6 vs. $16.6 \mathrm{yrs}, \mathrm{p}=0.01)$. BMD in the femoral neck but not in the spine was independently associated with a history of a $\mathrm{N}$ VF after adjusting for age, weight, height, disease duration and Larsen score using logistic regression.

\begin{tabular}{llll}
\multicolumn{4}{l}{ Abstract SAT0159 Table 1} \\
\hline No N-VF $(\mathbf{n}=124)$ & N-VF ( $\mathbf{n}=25)$ & P-value \\
\hline Age (yrs) & $60.4(5.8)$ & $64.8(3.9)$ & $<0.001$ \\
Larsen score & $37.2(29.5)$ & $59.2(34.2)$ & 0.002 \\
Fem.neck (BMD) & $0.84(0.09)$ & $0.79(0.06)$ & 0.009 \\
Spine L2-4 (BMD) & $1.10(0.10)$ & $1.06(0.07)$ & 0.06 \\
\hline
\end{tabular}

Conclusion Our cross sectional findings indicate that BMD is a risk factor for N-VF in female RA patients. Weather this concerns a causal relationship needs confirmation in prospective studies.

\section{SAT0160 THE EFFECT OF CALCITONIN ON PAIN AND QUALITY OF LIFE IN POSTMENOPAUSAL OSTEOPOROTIC WOMEN}

D Ofluoglu, G Akyuz, O Kayhan, O Umay. Marmara University Hospital, Department of Physical Medicine and Rehabilitation, Istanbul, Turkey

10.1136/annrheumdis-2001.647

Background Back pain is the most frequent symptom in osteoporosis.

Objectives The aim of this study was to investigate the effect of calcitonin on plasma beta-endorphin levels, pain and quality of life in postmenopausal women with osteoporosis.

Methods In our study, 100 IU salmon calcitonin with subcutaneous or placebo injection was given for 2 weeks and was continued with same dose of nasal spray or placebo for 3 months to postmenopausal women who had severe back pain due to osteoporosis. All patients took $1000 \mathrm{mg}$ elementary Calcium per day. We investigated the effectiveness of calcitonin on plasma betaendorphin levels, pain, and quality of life. There were thirty patients with a mean age of $58.17+5.36$ years in the treatment group and twenty-six patients with a mean age of $58.77+5.15$ years in the placebo group. Baseline plasma beta-endorphin levels were measured and repeated after two weeks and 3 months. Patients? pain was evaluated by visual analogue scale (VAS), and the modified face scale (MFS); and the quality of life was also assessed by Beck Depression Index (BDI) and Nottingham Health Profile (NHP) at the end of the second week and the third month.

Results We found that the measurements of the plasma betaendorphin level of the treatment group were significantly higher than those of the placebo group both at the end of the second week and the third month $(\mathrm{p}<0.001)$. Visual analogue scale, MFS, BDI and NHP scores were improved at the end of the second week in both groups $(p<0.05)$. However, the improvement of these scores decreased in three months in the placebo group. They did not change in the placebo group at the end of the 3rd month statistically ( $\mathrm{p}>0.05$ ).

Conclusion As a result, we suggest that calcitonin is a very strong analgesic agent via increasing the plasma beta-endorphin levels in postmenopausal osteoporosis and improves their physical activities and the quality of life due to decreasing back pain.

\section{REFERENCES}

1 Cantarelli FB, Szejnfeld VL, Oliveira LM, et al. Quality of life in patients with osteoporosis fractures: cultural adaptation, reliability and validity of the osteoporosis assessment questionnaire. Clin Exp Rheumatol. 1999;17(5):547-51

2 Civitelli R. Calcitonin. In: Marcus R, Feldman D, Kelsey J, eds. Osteoporosis. Sandiego/CA: Academic Press, 1996: 1235-58

3 Lyrýtýs GP, loannidis GV, Karachalios T, et al. Pain relief from nasal salmon calcitonin in osteoporotic vertebral crush fractures. A double blind, placebo-controlled clinical study. Acta Orthop Scand. 1997;68(Suppl 275):112-14

\section{SAT0161 MENATETRENONE ACTS DIRECTLY ON CIRCULATING HUMAN OSTEOCLAST PRECURSORS}

T Hirayama, H Taira, O Kudo, I Itonaga, T Torisu, Y Fujikawa. Department of Orthopaedic Surgery, Oita Medical University, Oita-Gun, Japan

\subsection{6/annrheumdis-2001.648}

Background Vitamin $\mathrm{K}$ is known to be an essential cofactor in the post-translational gamma-carboxylation of clotting factors. Since the gamma-carboxyglutamic acid-containing protein, osteocalcin, was discovered in bone matrix, vitamin $\mathrm{K}$ was thought to play a role in bone metabolism. Both in vitro and in vivo studies have shown that menatetrenone, a vitamin $\mathrm{K} 2$ homologue, can act directly on bone metabolism.

Objectives Although the relationship between menatetrenone and bone resorption appears clear, ${ }^{1,2}$ it is still not certain whether menatetrenone act directly on osteoclast precursors to influence their differentiation into osteoclasts. In this study, we have invetgated whether menatetrenone has a direct effect on circulating osteoclast precursors to influence osteoclast differentiation.

Methods Monocytes were cultured with osteoprotegerin ligand (OPGL) in the presence of macrophage stimulating factor (MCSF) on glass coverslips and dentine slices. Menatetrenone or vitamin $\mathrm{K} 1$ was then added to the cultures in $10-7,-6,-5$ (M) respectively. Geranylgeraniol or phytol (alcohol forms of the respective side chain of menatetrenone and vitamin $\mathrm{K} 1$ ) was also added to the cultures. After 7 and 14 days incubation, cultures were assessed for cytochemical and functional evidence of osteoclast differentiation.

Results The number of tartrate-resistant acid phosphatase (TRAP)positive multinucleated cells (MNCs) and the area of lacunar resorption were decreased when menatetrenone was added. Dosedependent inhibition of osteoclast formation and lacunar resorption was seen. Vitamin K1, however, did not inhibite the formation of TRAP positive MNCs nor lacunar resorption. The addition of geranylgeraniol in the cultures inhibited osteoclast formation in a dose dependent manner. Phytol did not affect the number of TRAP-positive MNCs nor lacunar resorption.

Conclusion Menatetrenone not only influence osteoclast formation in the presence of bone stromal cells but also act directly on circulating osteoclast precursors to influence osteoclast 